\title{
Ocorrência de Diaemus youngi (Jentink 1893), Chiroptera, no Estado do Rio de Janeiro
}

\author{
Luciana de Moraes Costa ${ }^{1,2}$; Débora Moraes de Oliveira ${ }^{1}$; \\ Agata Freitas Prata Dias e Fernandes ${ }^{1}$, Carlos Eduardo Lustosa Esberard ${ }^{I}$ \\ ${ }^{I}$ Instituto de Biologia Animal, Universidade Federal Rural do Estado do Rio de Janeiro - UFRRJ, \\ Km 47 da antiga Rio-São Paulo, CP 74503, CEP 23851-970, Seropédica, Rio de Janeiro, Brasil \\ ${ }^{2}$ Autor para correspondência: Luciana de Moraes Costa, e-mail: luciana_bio@terra.com.br, http://www.ufrrj.br
}

Costa, L. M.; Oliveira, D. M.; Dias e Fernandes, A. F. P.; Esberard, C. E. L. Occurrence of Diaemus youngi (Jentink 1893), Chiroptera, in the State of Rio de Janeiro. Biota Neotrop., vol. 8, no. 1, Jan./Mar. 2008. Available from: <http://www.biotaneotropica.org.br/v8n1/en/abstract?short-communication+bn00408012008>.

\begin{abstract}
Diaemus youngi is a vampire bat that feeds preferentially on bird blood. This bat is considered as the least frequent among the three hematofagous species throughout its geographical distribution. From 2000 to 2006 , surveys for vampire control were carried out in seven localities from the State of Rio de Janeiro where attacks to birds were reported by land owners. Bats were captured in mist nets opened during two or three nights in each locality, usually in the vicinity of attacked birds. A total of eight specimens of D. youngi were collected in the seven studied localities and two records from two other localities made by other researchers were confirmed for the State of Rio de Janeiro. Apparently, D. youngi prefers to prey on domestic and wild birds that rest on high perches. This bat species may present higher abundance and larger distribution range those described here, judging by the number of reports of attacks in commercial houses of ornamental birds that raise animals in semi-freedom in Southeastern Brazil.
\end{abstract}

Keywords: geographic distribution, white-winged vampire bats, bird attacks.

Costa, L. M.; Oliveira, D. M.; Dias e Fernandes, A. F. P.; Esberard, C. E. L. Ocorrência de Diaemus youngi (Jentink 1893), Chiroptera, no Estado do Rio de Janeiro. Biota Neotrop., vol. 8, no. 1, jan./mar. 2008. Disponível em: <http://www.biotaneotropica.org.br/v8n1/pt/abstract?short-communication+bn00408012008>.

Resumo: Diaemus youngi é um morcego hematófago obrigatório que se alimenta preferencialmente do sangue de aves. Este morcego é considerado o menos freqüiente entre as três espécies hematófagas ao longo de toda a sua distribuição geográfica. Entre 2000 e 2006, foram realizadas campanhas para a captura de morcegos hematófagos em sete localidades do Estado do Rio de Janeiro, onde ataques a aves haviam sido reportadas por proprietários de terra. Morcegos foram capturados em redes de neblina abertas durante duas ou três noites em cada localidade, geralmente próximas às aves atacadas. Um total de oito espécimes de $D$. youngi foi coletado nas sete localidades estudadas, e dois registros feitos por outros pesquisadores em outras duas localidades foram confirmados para o Estado do Rio de Janeiro. Diaemus youngi prefere, aparentemente, restringir seus ataques a aves domésticas e selvagens que apresentam o comportamento de se empoleirar. Esta espécie de morcego pode apresentar abundância e área de distribuição maiores do que as aqui descritas, a julgar pelo número de relatos de ataques em criatórios comerciais de aves ornamentais que criam animais em regime de semi-liberdade no Sudeste do Brasil.

Palavras-chave: distribuição geográfica, morcego hematófago de asas brancas, ataques a aves. 
Costa, L. M. et al.

\section{Introdução}

Diaemus youngi (Jentink 1893) é um morcego hematófago que se alimenta preferencialmente do sangue de aves (Gardner 1977, Uieda 1993, Greenhall \& Schutt 1996). Sua distribuição geográfica conhecida na América do Sul é da Bacia Amazônica ao nordeste da Argentina (Koopman 1993), tendo sido confirmada em 13 dos 26 estados brasileiros (Aguiar et al. 2006). É uma espécie rara em todo o território nacional (e.g. Aguiar et al. 2006) e em toda a sua distribuição geográfica (Greenhall \& Schutt 1996), fato que suscitou a sua inclusão nas listas de espécies ameaçadas de extinção do Estado do Rio de Janeiro (Bergallo et al. 2000) e do Paraná (Margarido \& Braga 2004). Até o momento sua ocorrência no Estado do Rio de Janeiro só era mencionada em publicações para o município de Barra Mansa, onde um exemplar foi capturado ao atacar galinhas empoleiradas em parreira de chuchu (Peracchi \& Albuquerque 1971), e em Carmo, onde Avilla et al. (2001) registraram a espécie.

Ao realizarmos coletas para controle de morcegos hematófagos no Estado do Rio de Janeiro, confirmamos a ocorrência desse morcego em outras localidades, como relatado a seguir.

\section{Material e Métodos}

Entre 2000 e 2006, foram realizadas campanhas para a captura de morcegos hematófagos que atacam aves em sete localidades do Estado do Rio de Janeiro (Figura 1, Tabela 1). Estes locais foram escolhidos entre os casos de ocorrência de ataques informados pelos proprietários à Fundação RIOZOO, ao Instituto Brasileiro de Meio Ambiente e Recursos Renováveis ou diretamente aos autores. Foram escolhidos os locais cujos proprietários se prontificaram a cobrir os custos das coletas e que relataram a morte de aves exóticas.

Os morcegos foram capturados com redes de neblina abertas durante duas ou três noites junto aos animais atacados. As noites de

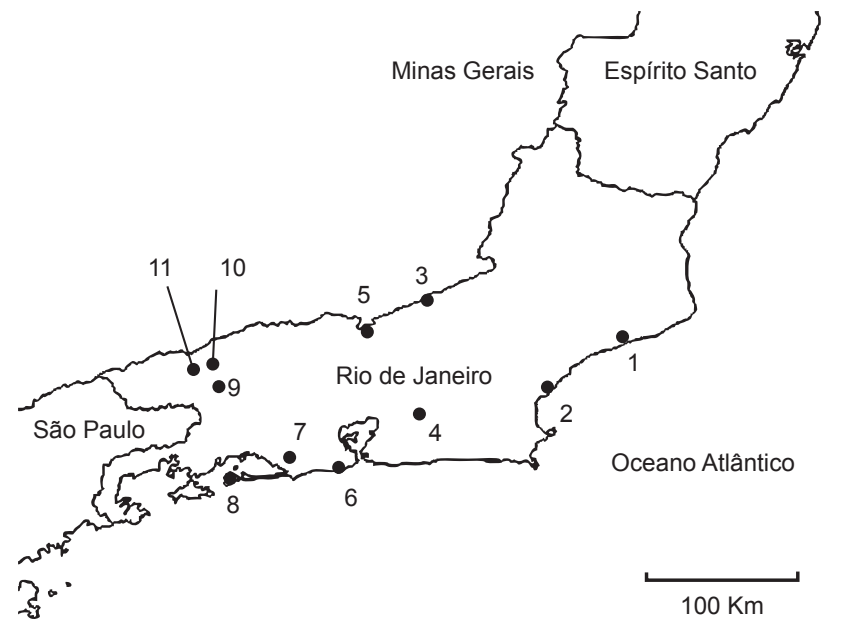

Figura 1. Localidades de captura de Diaemus youngi no Estado do Rio de Janeiro. 1) Parque Nacional Restinga de Jurubatiba, Quissamã (Bergallo et al. 2004), 2) Morro de São João, Casimiro de Abreu, 3) Carmo (Avilla et al 2001), 4) Parada Modelo, Guapimirim, 5) Bemposta, Três Rios, 6) Barra da Tijuca, Cidade do Rio de Janeiro, 7) Barra de Guaratiba, Cidade do Rio de Janeiro, 8) Ilha da Marambaia, Mangaratiba, 9) Barra Mansa (Peracchi \& Albuquerque 1971), 10) Volta Redonda (dados de R. Pineschi e colaboradores), e 11) Refúgio de Vida Silvestre, Valença.

Figure 1. Localities of capture of Diaemus youngi in the State of Rio de Janeiro. 1) Parque Nacional Restinga de Jurubatiba, Quissamã (Bergallo et al. 2004), 2) Morro de São João, Casimiro de Abreu, 3) Carmo (Avilla et al 2001), 4) Parada Modelo, Guapimirim, 5) Bemposta, Três Rios, 6) Cidade do Rio de Janeiro, 7) Barra de Guaratiba, Cidade do Rio de Janeiro, 8) Ilha da Marambaia, Mangaratiba, 9) Barra Mansa (Peracchi \& Albuquerque 1971), 10) Volta Redonda (dados de R. Pineschi e colaboradores), and 11) Refúgio de Vida Silvestre, Valença.

Tabela 1. Localidade, município, coordenadas, data, sexo, peso e comprimento do antebraço dos exemplares de Diaemus youngi analisados pelos autores no Estado do Rio de Janeiro de 2000 a 2006 e disponíveis na bibliografia. Os números indicam a posição no mapa da Figura 1.

Table 1. Locality, municipality, coordinates, date, sex, weight and length of the forearm of the specimens of Diaemus youngi analyzed by the authors in the State of Rio de Janeiro from 2000 to 2006 and available in the literature. The numbers indicate the position in the map of Figure 1.

\begin{tabular}{|c|c|c|c|c|c|c|}
\hline No. & Localidade & Coordenadas e altitude & Data & Sexo & Peso (g) & Antebraço (mm) \\
\hline 1 & $\begin{array}{l}\text { Parque Nacional Restinga } \\
\text { de Jurubatiba, Quissamã }\end{array}$ & $\begin{array}{l}41^{\circ} 28^{\prime} 12,0^{\prime \prime} \mathrm{W} \text { e } 22^{\circ} 06^{\prime} 14,4^{\prime \prime} \mathrm{S}, \\
\text { altitude } 5 \mathrm{~m} \text {, } \\
\text { coletores R. Mangolin, D. Raices e M.A.R. Mello }\end{array}$ & 2001 & $\mathrm{M}$ & - & - \\
\hline 2 & $\begin{array}{l}\text { Morro de São João, } \\
\text { Casimiro de Abreu }\end{array}$ & $\begin{array}{l}42^{\circ} 00^{\prime} 34,8^{\prime \prime} \mathrm{W} \text { e } 22^{\circ} 31^{\prime} 13,8^{\prime \prime} \mathrm{S} \text {, } \\
\text { altitude } 40 \mathrm{~m}\end{array}$ & 06/10/01 & $\mathrm{F}$ & 44,3 & 52,95 \\
\hline 2 & $\begin{array}{l}\text { Morro de São João, } \\
\text { Casimiro de Abreu }\end{array}$ & $\begin{array}{l}42^{\circ} 00^{\prime} 34,8^{\prime \prime} \mathrm{W} \text { e } 22^{\circ} 31^{\prime} 13,8^{\prime \prime} \mathrm{S} \text {, } \\
\text { altitude } 40 \mathrm{~m}\end{array}$ & $25 / 11 / 01$ & M & 41,8 & 51,66 \\
\hline 3 & Carmo & Fonte: Avilla et al. 2001 & & & & \\
\hline 4 & $\begin{array}{l}\text { Parada Modelo, } \\
\text { Guapimirim }\end{array}$ & $\begin{array}{l}42^{\circ} 55^{\prime} 70,5^{\prime} " \mathrm{~W} \text { e } 22^{\circ} 33^{\prime} 24,2^{\prime \prime} \mathrm{S} \text {, } \\
\text { altitude } 70 \mathrm{~m}\end{array}$ & $16 / 09 / 01$ & $\mathrm{~F}$ & 44,7 & 52,70 \\
\hline 5 & Bemposta, Três Rios & $\begin{array}{l}43^{\circ} 12^{\prime} 19,8^{\prime \prime} \mathrm{W} \text { e } 22^{\circ} 07^{\prime} 00,12^{\prime \prime} \mathrm{S} \text {, } \\
\text { altitude } 390 \mathrm{~m}\end{array}$ & $08 / 09 / 00$ & $\mathrm{~F}$ & 38,0 & 55,00 \\
\hline 6 & $\begin{array}{l}\text { Barra da Tijuca, } \\
\text { Cidade do Rio de Janeiro }\end{array}$ & $\begin{array}{l}43^{\circ} 17^{\prime} 48,9^{\prime \prime} \mathrm{W} \text { e } 23^{\circ} 00^{\prime} 21,8^{\prime \prime} \mathrm{S} \text {, } \\
\text { altitude } 45 \mathrm{~m}\end{array}$ & $27 / 10 / 01$ & M & 38,6 & 52,50 \\
\hline 7 & $\begin{array}{l}\text { Barra de Guaratiba, } \\
\text { Cidade do Rio de Janeiro }\end{array}$ & $\begin{array}{l}43^{\circ} 32^{\prime} 21,2^{\prime \prime} \mathrm{W} \text { e } 22^{\circ} 59^{\prime} 44,6^{\prime \prime} \mathrm{S} \text {, } \\
\text { altitude } 50 \mathrm{~m}\end{array}$ & $21 / 10 / 00$ & $\mathrm{~F}$ & 41,0 & 51,21 \\
\hline 8 & Barra Mansa & Fonte: Peracchi, A.L. \& Albuquerque, S.T. 1971 & & & & \\
\hline 9 & $\begin{array}{l}\text { Ilha da Marambaia, } \\
\text { Mangaratiba }\end{array}$ & $\begin{array}{l}43^{\circ} 57^{\prime} 56,2^{\prime \prime} \mathrm{W} \text { e } 22^{\circ} 04^{\prime} 25,7^{\prime \prime} \mathrm{S} \text {, } \\
\text { altitude } 75 \mathrm{~m}\end{array}$ & $25 / 11 / 06$ & M & 41,8 & 55,10 \\
\hline 10 & Volta Redonda & Coletores R. Pineschi e B. Costa & $11 / 04 / 01$ & M & 43,0 & 53,42 \\
\hline 11 & $\begin{array}{l}\text { Refúgio da Vida Silvestre, } \\
\text { Valença }\end{array}$ & $\begin{array}{l}44^{\circ} 16^{\prime} 03,9^{\prime \prime} \mathrm{W} \text { e } 22^{\circ} 22^{\prime} 47,8^{\prime \prime} \mathrm{S} \text {, } \\
\text { altitude } 600 \mathrm{~m}\end{array}$ & 05/04/05 & $\mathrm{F}$ & 42,0 & 53,63 \\
\hline
\end{tabular}


coleta foram agendadas para que pelo menos uma fosse realizada na fase clara (lua cheia e crescente) e outra na fase escura (lua nova e minguante) do ciclo lunar. As redes permaneceram abertas por toda a noite e inspecionadas em intervalos de 20 minutos. Os morcegos capturados foram identificados, mensurados e marcados com coleiras plásticas providas de cilindros coloridos (Esbérard \& Daemon 1999). Após esses procedimentos eram soltos, exceto por um ou dois exemplares de cada espécie, que foram sacrificados com o uso de éter, fixados em formol a 4\%, estocados em etanol a 70\% e tombados como material testemunho na coleção de referência do Projeto Morcegos Urbanos, atualmente lotada na Universidade Federal Rural do Rio de Janeiro (IBAMA processo 1755/89), bem como todos os exemplares de Desmodus rotundus (E. Geoffroy, 1810), que foram sacrificados para minimizar os ataques a seres humanos e animais domésticos.

\section{Resultados}

Nas sete localidades amostradas no Estado do Rio de Janeiro durante este estudo, foram coletados oito exemplares de Diaemus youngi (Tabela 1). Além destes registros, um exemplar de D. youngi foi capturado longe de criações de aves no Parque Nacional Restinga de Jurubatiba (Bergallo et al. 2004). Este exemplar foi identificado em campo como Diphylla ecaudata Spix, 1823, mas a fotografia tirada pelos coletores (R. Mangolin, D.S.L. Raíces e M.A.R. Melo - UERJ) não deixa dúvidas sobre sua identificação correta. Recebemos também um exemplar capturado por Renato Pineschi e Benedito Costa Neves (Curso de Pós-Graduação da UFRuralRJ), em propriedade próxima a Reserva da Cicuta, Volta Redonda, ao atacar aves domésticas empoleiradas junto a galinheiro, onde foi constatado o ataque simultâneo por D. rotundus e D. ecaudata. Neste local, foi encontrada em 09/10/02, uma colônia de D. ecaudata refugiada em um porão, composta por cinco machos e cinco fêmeas.

Dois exemplares de D. youngi foram capturados em Casimiro de Abreu com redes armadas no interior de cercado de aves. Estes foram capturados ao atacarem pavões azuis (Pavo cristatus Linnaeus, 1758) e perus (Meleagris gallopavo Linnaeus, 1758) empoleirados em imbaúbas (Cecropia glaziovi, Cecropiaceae) e mangueiras (Mangifera indica Linnaeus, Anacardiaceae) a cerca de $4 \mathrm{~m}$ de altura. Várias mortes de aves ornamentais foram relatadas neste local até a captura destes exemplares, sem ser observada a presença de outras espécies de morcegos hematófagos. No entanto, em pastos próximos (cerca de $400 \mathrm{~m}$ ), constatamos, através de captura com redes de neblina elevada densidade de $D$. rotundus atacando eqüinos, ovinos e bovinos. Emas [Rhea americana (Linnaeus, 1758)] aparentemente não foram atacadas, apesar de mantidas em cercado contíguo ao das aves atacadas.

Em Guapimirim, um ataque de morcegos hematófagos foi relatado em um criatório de aves, onde mortes foram observadas entre galinhas domésticas [Gallus gallus (Linnaeus, 1758)], galinhas-d'angola [Numidia meleagris (Linnaeus, 1758)], perus e pavões azuis por vários anos antes da realização da primeira coleta. Redes armadas entre as árvores onde pernoitavam as galinhas-d'angola, a cerca de $4 \mathrm{~m}$ de altura, resultaram na captura de um exemplar às 03:30 horas. Coletas realizadas em outras datas confirmaram posteriormente a presença também de D. ecaudata.

Em Três Rios, um exemplar foi capturado junto a um galinheiro às 00:00 horas ao se aproximar de um pavão azul que dormia no interior de um cercado, a cerca de $2 \mathrm{~m}$ de altura, junto com galinhas domésticas.

Um exemplar foi capturado às 02:10 horas, após atacar um pavão azul empoleirado em mangueira a mais de $5 \mathrm{~m}$ de altura em um criatório científico de aves silvestres e domésticas na Cidade do Rio de Janeiro, limítrofe ao Parque Nacional da Tijuca (3300 ha de floresta secundária). Neste local, numerosas mortes entre galinhasd'angola vulturinas [Acryllium vulturinum (Hardwicke, 1834)] antes da captura. A continuidade das coletas revelou também a presença de D. ecaudata no local.

Em criatório científico de aves localizado em Barra de Guaratiba, Cidade do Rio de Janeiro, longe de área florestada (mais de $5 \mathrm{~km}$ ), capturamos exemplar às 04:30 horas, enquanto atacava galinhasd'angola vulturinas que dormiam empoleiradas em árvore a cerca de 3,5 $\mathrm{m}$ do solo. Neste local o ataque cessou até o fim do primeiro semestre de 2001, quando recomeçou em vulturinas, pavões verdes (Pavo muticus Linnaeus, 1766) e pavões azuis.

Longe de criações de aves, um exemplar foi capturado na Ilha da Marambaia, Município de Mangaratiba, às 22:00 horas, em redes armadas sobre dique de represa, em borda de mata secundária.

Em Valença durante 12 minutos foi observado um morcego hematófago ingerindo sangue que vertia da região da cloaca de uma galinha doméstica empoleirada a cerca de $6 \mathrm{~m}$ de altura em uma jabuticabeira (Myrciaria cauliflora Berg., Myrtaceae) às 00:10 horas, sem ser possível sua identificação segura. Um exemplar de $D$. youngi foi capturado com rede armada no nível do solo, a cerca de 1,5 m de distância da ave atacada.

\section{Discussão}

Os dados aqui apresentados sugerem que Diaemus youngi ataca preferencialmente aves domésticas e selvagens que apresentam o comportamento de se empoleirar a alturas superiores a $3 \mathrm{~m}$ (66,67\% dos ataques, média de 3,28 $\pm 1,9 \mathrm{~m}, \mathrm{n}=9$ ), mas pode atacar também aves próximas ao solo. De fato, Diaemus youngi aparentemente é mais especializada para atacar aves enquanto empoleiradas, pendurando-se no galho onde pernoita a ave (Uieda 1993, Schutt et al. 1999). No entanto, observações em cativeiro demonstram que este morcego também realiza ataques enquanto pousados no substrato da gaiola (Schutt et al. 1999).

Diaemus youngi pode estar se beneficiando da prática de alguns criadores de aves ornamentais de manter exemplares em regime de semi-liberdade, principalmente pavões e galinhas-d'angola. A frequiência de ocorrência dessa espécie de morcego pode ser ainda maior que a já conhecida, a julgar pelo número de relatos de ataques em condições similares em criatórios comerciais de aves ornamentais, como, por exemplo, em Miguel Pereira, Paty do Alferes e na Cidade do Rio de Janeiro. No entanto, taxas de mortalidade mais altas entre aves ornamentais e domésticas foram relatadas em localidades onde a presença de D. youngi ocorreu simultaneamente à D. ecaudata e D. rotundus. A maior mortalidade decorrente do ataque de D. rotundus e D. ecaudata deve-se ao maior tamanho da mordedura e aos repetitivos ataques na mesma noite à mesma ave por estas espécies (Uieda 1993).

A inclusão de D. youngi em duas listas regionais de espécies ameaçadas de extinção (Bergallo et al. 2000, Margarido \& Braga 2004) foi motivada principalmente pela sua suposta raridade e a possibilidade da mesma sofrer extermínio pelas técnicas atuais de controle de morcegos hematófagos, principalmente devido ao despreparo dos técnicos envolvidos, que podem ter dificuldade na identificação da espécie, aplicando pasta vampiricida nesses exemplares, como nos de D. rotundus (Aguiar et al. 2006). Das 11 localidades onde D. youngi foi registrada no Estado do Rio de Janeiro, em apenas três a espécie estaria protegida, sendo um Parque Nacional (Parque Nacional Restinga de Jurubatiba), uma Reserva Particular do Patrimônio Natural (Morro de São João) e uma área administrada pela Marinha do Brasil (Ilha da Marambaia). Além disso, a maior parte das localidades confirmadas para a espécie no Estado do Rio de Janeiro situa-se próxima a remanescentes de mata $(66,67 \%$ das capturas). Esta espécie tem sido relatada para áreas abertas, florestas, quintais e pastagens (Greenhall \& Schutt 1996), sem, contudo, ter sido quantificado o total de capturas em cada ambiente. 
Costa, L. M. et al.

As capturas no Estado do Rio de Janeiro aqui relatadas ocorreram desde o nível do mar a até $600 \mathrm{~m}$ de altitude (média de altitude das localidades 189,44 $\pm 208,21 \mathrm{~m}, \mathrm{n}=9$ ). Como a espécie distribui-se até o nordeste da Argentina (Koopman 1993), onde o clima é subtropical, o registro do limite altitudinal desta espécie pode elevar-se com a realização de coletas em maiores altitudes. Além disso, as capturas foram mais freqüentes após as 00:00 horas $(n=9)$, sugerindo que é necessário amostrar por toda a noite para maior sucesso na captura desta espécie. Os oito exemplares coletados em 2004 no bioma do Cerrado por Aguiar et al. (2006), ao saírem de caverna, foram capturados entre 20:00 horas e 23:00 horas em redes armadas até as 00:00 horas, o que sugere um início mais tardio da atividade fora do refúgio do que nas demais espécies de morcegos hematófagos (veja Uieda 1993). As capturas no presente trabalho foram realizadas junto às presas atacadas, e a diferença no horário de captura deve incluir o tempo necessário para o morcego abandonar o refúgio, deslocar-se até o local de repouso da presa, reconhecer o local com vôos próximos e aproximar-se desta para a realização da mordedura (Uieda 1993).

Assim sendo, sugerimos que é necessário incrementar o esforço de captura de morcegos vampiros junto aos criadores de aves ornamentais para que a distribuição e densidade de $D$. youngi seja melhor descrita e seu status de conservação melhor avaliado.

\section{Agradecimentos}

Instituto Biomas, UERJ e CNPq (processo 152910/2004-0) pelo financiamento do trabalho de campo em Valença e a UFRuralRJ pelo trabalho de campo na Ilha da Marambaia; aos Criatórios Fazano, Castilho, Fazenda Paciência, Condomínio Paraíso e Fazenda Carioca Engenharia pela permissão para coletas e custeio do trabalho; Marco A. R. Mello cedeu gentilmente a fotografia e os dados do exemplar capturado no Parque Nacional Restinga de Jurubatiba; Renato Pineschi doou o exemplar capturado em Volta Redonda. Coletas realizadas com licença especial para captura (Processos 1785/89-IBAMA e 4156/95-46 AC-SUPES/DF/IBAMA). Esta contribuição faz parte da dissertação de mestrado de L.M. Costa.

\section{Referências Bibliográficas}

AGUIAR, L.M.S., CAMARGO, W.R. \& PORTELLA, A.S. 2006. Occurrence of white-winged vampire bat, Diaemus youngi (Mammalia, Chiroptera), in the Cerrado of Distrito Federal, Brazil. Revista Brasileira de Zoologia 23(3):893-896.
AVILLA, L.S., ROZENSZTRANCH, A.M.S. \& ABRANTES, E.A.L. 2001. First record of the South American Flat-Headed Bat Neoplatymops mattogrossensis (Vieira, 1942) in Southeastern Brazil (Chiroptera, Molossidae). Boletim do Museu Nacional. Rio de Janeiro 463:1-6.

BERGALLO, H.G., GEISE, L., BONVICINO, C.R., CERQUEIRA, R., D'ANDREA. P.S., ESBERÁRD, C.E.L., FERNANDEZ, F.A.S., GRELLE, C.E., PERACCHI, A., SICILIANO, S. \& VAZ, S.M. 2000. Mamíferos. p. 125-135. In: Bergallo, H.G., Rocha, C.F.D., Alves, M.A.S. \& Sluys, M.V. (Eds.) A fauna ameaçada de extinção do Estado do Rio de Janeiro, p.125-136. Editora da UERJ, Rio de Janeiro, 166p.

BERGALLO, H.G., F. MARTINS-HATANO, D.S.L. RAÍCES, T.T.L. RIBEIRO, A.G. ALVES, J.L. LUZ, R. MANGOLIN \& M.A.R. MELLO. 2004. Os mamíferos da Restinga de Jurubatiba. Pp. 215-230. In C.F.D. Rocha, F.A. Esteves \& F.R. Scarano (Eds.), Pesquisas de Longa Duração na Restinga de Jurubatiba - Ecologia, história natural e conservação. Rima Editora, São Carlos, 374p.

ESBÉRARD C.E.L. \& DAEMON, C. 1999. Novo método para marcação de morcegos. Chiroptera Neotropical 5(1-2):116-117.

GARDNER, A.L. 1977. Feeding habits. In R.J. Baker, J.K. Jones Jr \& D.C. Carter (Eds.). Biology of bats of the New World family Phyllostomidae. Part II. Special Publications, Texas Tech University 13:1-64.

GREENHALL, A.M. \& SCHUTT JR, W.A. 1996. Diaemus youngi. Mammalian Species 533:1-7.

KOOPMAN, K.F. 1993. Order Chiroptera. In Wilson, D. E. \& D. M. Reeder (eds.). Mammals species of the world: A taxonomic and geographic reference. 2a. ed. Smithsonian Institution, Washinghton, D.C, p. 137-232.

MARGARIDO, T.C.C. \& BRAGA, F.C. 2004. Mamíferos, p. 27-142. In: S.B. MIKICH \& R.S. BÉRNILS (Eds). Livro vermelho da fauna ameaçada no Estado do Paraná. Curitiba. Instituto Ambiental do Paraná, 763p.

PERACCHI, A.L. \& ALBUQUERQUE, S.T. 1971. Lista provisória dos quirópteros dos Estados do Rio de Janeiro e Guanabara, Brasil (Mammalia: Chiroptera). Revista Brasileira de Biologia 31(3):405-413.

SCHUTT, W.A. JR., MURADALI, F., MONDOL, N., JOSEPH, K. \& BROCKMANN, K. 1999. Behavior and maintenance of captive white-winged vampire bats, Diaemus youngi. Journal of Mammalogy 80(1):71-81.

UIEDA, W. 1993. Comportamento alimentar do morcego hematófago Diaemus youngi, em aves domésticas. Revista Brasileira de Biologia 53(4):529-538. 